EPJ Web of Conferences 60, 08004 (2013)

DOI: $10.1051 /$ epjconf/20136008004

(C) Owned by the authors, published by EDP Sciences, 2013

\title{
Electroweak theory and global fit
}

\author{
M. Ciuchini ${ }^{1, a}$, E. Franco ${ }^{2, b}$, S. Mishima ${ }^{2,3, c}$, and L. Silvestrini2,d \\ ${ }^{1}$ INFN, Sezione di Roma Tre, Via della Vasca Navale 84, I-00146 Roma, Italy \\ ${ }^{2}$ INFN, Sezione di Roma, Piazzale A. Moro 2, I-00185 Roma, Italy \\ ${ }^{3}$ Dipartimento di Fisica, Università di Roma "La Sapienza", Piazzale A. Moro 2, I-00185 Roma, Italy
}

\begin{abstract}
We perform the fit of electroweak precision observables within the Standard Model with a $126 \mathrm{GeV}$ Higgs boson, compare the results with the theoretical predictions and discuss the impact of recent experimental and theoretical improvements. We introduce New Physics contributions in a model-independent way and fit for the $S, T$ and $U$ parameters and for a modified Higgs coupling to vector bosons. We point out that composite Higgs models are very strongly constrained. Finally, we compute the bounds on dimension-six operators relevant for the electroweak fit.
\end{abstract}

Electroweak Precision Observables (EWPO) have played a key role in constraining New Physics (NP) for the past twenty years [1-11]. The most striking examples of the power of these indirect constraints are the prediction of the top and Higgs masses. The experimental situation improved dramatically in the past year, with the precise measurement of the Higgs mass at the LHC $[12,13]$. In addition, the information on other key Standard Model (SM) parameters such as the top and $W$ boson masses has increased considerably, leading altogether to a sizable progress in the electroweak (EW) fit. It is therefore phenomenologically relevant to reassess the constraining power of the EW fit in the light of these recent experimental improvements. To this aim, we perform the EW fit in the SM and update the constraints on oblique NP. On the theory side, the full two-loop fermionic EW contributions to the $R_{b}^{0}$ observable have been recently numerically calculated in ref. [14]. The implementation of this result in the global fit has a large impact but represents a nontrivial problem, as we illustrate in detail below. A very interesting question that can be tackled with present data is whether the Higgs boson is elementary or composite. Using a general effective Lagrangian for Higgs boson interactions [15-18], we analyze the constraints on the Higgs coupling to vector bosons, and find out that this coupling can be determined from the fit with an uncertainty of 5\% at $95 \%$ probability, while much larger departures from the SM value are expected in generic composite Higgs models. Thus, the EW fit points to an elementary Higgs or to composite Higgs models in which additional contributions are present to restore the agreement with EWPO. Finally, we consider the most general effective Lagrangian relevant

\footnotetext{
a e-mail: Marco.Ciuchini@ $@$ roma3.infn.it

be-mail: Enrico.Franco@roma1.infn.it

ce-mail: Satoshi.Mishima@roma1.infn.it

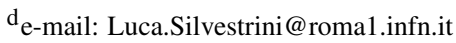

for EWPO and compute the constraints on the coefficients of dimension six operators, which can be translated into lower bounds on the NP scale assuming a given value for the couplings.

The part of the SM Lagrangian relevant for the computation of EWPO can be defined in terms of the following free parameters: the fine structure constant $\alpha$, the muon decay constant $G_{\mu}$, the $Z$ boson mass $M_{Z}$, the strong coupling $\alpha_{s}\left(M_{Z}^{2}\right)$, the top quark mass $m_{t}$ and the Higgs mass $m_{h}$. In addition, we introduce the effective parameter $\Delta \alpha_{\text {had }}^{(5)}\left(M_{Z}^{2}\right)$ to take into account the hadronic contribution to the running of $\alpha$. In terms of the seven parameters above, the SM prediction for all other EWPO can be computed. The recent measurements of $m_{h}$ by the ATLAS and CMS experiments are given by

$$
m_{h}= \begin{cases}125.5 \pm 0.2(\text { stat })_{-0.6}^{+0.5} \text { (syst) GeV } & \text { ATLAS } \\ 125.7 \pm 0.3 \text { (stat) } \pm 0.3 \text { (syst) GeV } & \text { CMS }\end{cases}
$$

We adopt the average $m_{h}=125.6 \pm 0.3 \mathrm{GeV}$ in the current study. According to ref. [19, 20], the world average of $\alpha_{s}\left(M_{Z}^{2}\right)$ from the fit to various data, excluding the EW precision measurements, is given by $\alpha_{s}\left(M_{Z}^{2}\right)=$ $0.1184 \pm 0.0006$. For the hadronic contribution to the running of the electromagnetic coupling, we adopt the recent evaluation $\Delta \alpha_{\text {had }}^{(5)}\left(M_{Z}^{2}\right)=0.02750 \pm 0.00033$ in ref. [21]. In the absence of a world average for the top pole mass, we adopt the Tevatron average $m_{t}=173.18 \pm 0.56$ (stat) \pm 0.75 (syst) $\mathrm{GeV}=173.2 \pm 0.9 \mathrm{GeV}$ [22], fully compatible with the LHC result $m_{t}=173.3 \pm 0.5$ (stat) \pm 1.3 (syst) $\mathrm{GeV}$ [23]. The renormalization group runnings of the strong coupling constant and the fermion masses are taken into account up to three-loop level [24-26]. The measurement of the $Z$ boson mass is taken from LEP: $M_{Z}=$ $91.1875 \pm 0.0021 \mathrm{GeV}$ [27]. Finally, the parameters $G_{\mu}$ and $\alpha$ are fixed to be constants: $G_{\mu}=1.1663787 \times 10^{-5}$ $\mathrm{GeV}^{-2}$ and $\alpha=1 / 137.035999074$, respectively [19]. 
The SM contributions to the EWPO have been calculated very precisely including higher-order radiative corrections. For a recent discussion of the theoretical status of EWPO we refer the reader to ref. [28]; here we focus on the discussion of the calculation of fermionic twoloop corrections to the $Z b \bar{b}$ vertex. The complete two-loop formulæ for the coupling $\rho_{Z}^{f}$ are currently missing. Recently, the complete fermionic two-loop EW corrections have been calculated for $R_{b}^{0}=\Gamma_{b} / \Gamma_{h}$ in ref. [14], where an approximate formula has been presented. However, from this approximate formula alone we cannot extract the values of $\rho_{Z}^{f}$ including fermionic two-loop corrections, that are necessary to compute other $\rho_{Z}^{f}$-dependent observables such as $R_{\ell}^{0}, R_{c}^{0}, \Gamma_{Z}$ and the hadronic cross section. The authors of ref. [14] have kindly provided us with the approximate formulæ for $\Gamma_{u} / \Gamma_{b}$ and $\Gamma_{d} / \Gamma_{b}$ [29], which allow us to use the experimental information on one more observable in addition to $R_{b}^{0}$. To illustrate the impact of these two-loop corrections, we present our results for the $\mathrm{SM}$ fit in two scenarios. First, we use only the previously known leading and (where available) next-to-leading twoloop EW contributions of $O\left(G_{\mu}^{2} m_{t}^{4}\right)$ and $O\left(G_{\mu}^{2} m_{t}^{2} M_{Z}^{2}\right)$ in the large- $m_{t}$ expansion, together with the leading threeloop corrections of $O\left(G_{\mu}^{2} \alpha_{s} m_{t}^{4}\right)$ and $O\left(G_{\mu}^{3} m_{t}^{6}\right)$. Second, we use the approximate formulæ for $\Gamma_{u} / \Gamma_{b}$ and $\Gamma_{d} / \Gamma_{b}$ adding three free parameters to the fit, which represent the unknown corrections to $\rho_{Z}^{v}, \rho_{Z}^{\ell}$ and $\rho_{Z}^{b}$. The corrections to $\rho_{Z}^{u, d}$ can then be determined using the formulæ for $\Gamma_{u} / \Gamma_{b}$ and $\Gamma_{d} / \Gamma_{b}$. This is the optimal use we can make of the presently available theoretical information. It will be interesting to compare the fitted values of $\delta \rho_{Z}^{v}, \delta \rho_{Z}^{\ell}$ and $\delta \rho_{Z}^{b}$ with the theoretical expressions, once these will be available. As we shall see below, the corrections computed in ref. $[14,29]$ are surprisingly large, so that an independent check of the computation would be very useful.

The latest Tevatron average of the $W$-boson mass is $M_{W}=80.385 \pm 0.015 \mathrm{GeV}$ [30]. We use the results for $\Gamma_{Z}$, $\sigma_{h}^{0}, P_{\tau}^{\mathrm{pol}}, \mathcal{A}_{f}, A_{\mathrm{FB}}^{0, f}$ and $R_{f}^{0}$ from SLD/LEP-I $[27,31]$ and $\Gamma_{W}$ from LEP-II/Tevatron [32]. All experimental inputs are summarized in the second column of table 1, where we take into account the correlations among the inputs that can be found in ref. [31].

In the third column of table 1 we present the results of the SM fit obtained using the top pole mass and the expressions for $\Gamma_{u} / \Gamma_{b}$ and $\Gamma_{d} / \Gamma_{b}$ from refs. [14, 29]. As discussed above, in this case we do not have enough information to compute $\Gamma_{Z}, \sigma_{h}^{0}$ and $R_{\ell}^{0}$ at the same level of accuracy of $R_{b}^{0}$ and $R_{c}^{0}$. We therefore add three free parameters to the fit, representing the fermionic two-loop corrections $\delta \rho_{Z}^{v}, \delta \rho_{Z}^{\ell}$ and $\delta \rho_{Z}^{b}$. These parameters affect only the observables $\Gamma_{Z}, \sigma_{h}^{0}$ and $R_{\ell}^{0}$. In this way, while we cannot predict $\Gamma_{Z}, \sigma_{h}^{0}$ and $R_{\ell}^{0}$, we obtain a posterior for the parameters $\delta \rho_{Z}^{v}, \delta \rho_{Z}^{\ell}$ and $\delta \rho_{Z}^{b}$, which can be compared to the theoretical expressions once they become available. The other parameters $\delta \rho_{Z}^{u}$ and $\delta \rho_{Z}^{d}$ are determined from $\delta \rho_{Z}^{b}$ through $\Gamma_{u} / \Gamma_{b}$ and $\Gamma_{d} / \Gamma_{b}$, respectively. Notice that fits performed using the formula for $R_{b}^{0}$ from ref. [14] and the formulæ for $\rho_{Z}^{f}$ from ref. [34] are inconsistent, since the change in $R_{b}^{0}$ implies a change in $R_{c, \ell}^{0}, \Gamma_{Z}$ and $\sigma_{h}^{0}$. Furthermore, the
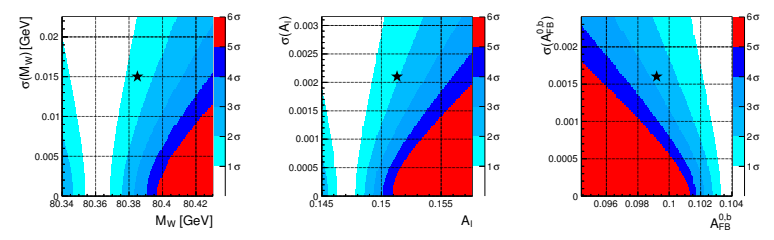

Figure 1. Compatibility plots of $M_{W}, \mathcal{A}_{\ell}$ and $A_{\mathrm{FB}}^{0, b}$. Any direct measurement corresponds to a point in the (central value, experimental error) plane, and its compatibility with the indirect determination is given in numbers of standard deviations by the color coding. The present experimental result is indicated by a star.

results of ref. [14] imply much larger two-loop fermionic corrections than expected from the expansion in ref. [34]. In fact, we can estimate the size of the unknown two-loop corrections as follows:

$\delta \rho_{Z}^{q}-\delta \rho_{Z}^{b} \approx \frac{\Gamma_{q} / \Gamma_{b}-\Gamma_{q}^{\prime} / \Gamma_{b}^{\prime}}{\Gamma_{q}^{\prime} / \Gamma_{b}^{\prime}}= \begin{cases}4.8 \times 10^{-3} & \text { for } q=u, \\ 4.4 \times 10^{-3} & \text { for } q=d,\end{cases}$

where $\Gamma_{f}\left(\Gamma_{f}^{\prime}\right)$ denotes a partial width including (omitting) the contribution from $\delta \rho_{Z}^{f}$, and the approximation $\rho_{Z}^{f} \approx 1$ has been used. Since these corrections are comparable in size to one-loop contributions, it would be desirable to have an independent confirmation of the calculation of ref. [14].

From the fit we also obtain posteriors for the SM parameters $\alpha_{s}\left(M_{Z}^{2}\right), \Delta \alpha_{\text {had }}^{(5)}\left(M_{Z}^{2}\right), M_{Z}, m_{t}$ and $m_{h}$ (see table 1).

To show the impact on the fit of the new calculation of $R_{b}^{0}$ [14], we present in table 2 the results obtained using instead refs. [34-40] for the leading and next-to-leading terms in the large- $m_{t}$ expansion for two-loop fermionic EW corrections to $\rho_{Z}^{f}$. As can be seen by comparing with the full results, the tension in $R_{b}^{0}$ is reduced. Notice that the indirect determination of $\alpha_{s}\left(M_{Z}^{2}\right)$ is much more precise in this case since we are not considering large unknown fermionic corrections to $\rho_{Z}^{f}$.

Let us now discuss the compatibility of the SM prediction with experimental data. To this aim, we use the compatibility plots introduced in ref. [33], where the difference in standard deviations between the fit prediction and the experimental result is given by the color coding.

The compatibility of $M_{W}, \mathcal{A}_{\ell}$ and $A_{\mathrm{FB}}^{0, b}$ is shown in figure 1. While these results are stable against the inclusion of the recently calculated two-loop fermionic corrections to $R_{b}^{0}$, the compatibility of $R_{b}^{0}$ is worsened by the inclusion of the results in ref. [14], as can be seen by comparing the plots in figure 2 .

Our numerical results agree with those obtained using the ZFITTER package [41-44]. Our fit results are compatible with the ones obtained by the LEP Electroweak Working Group [32] and also with the ones in refs. [45, 46]. A comparison with the recent Gfitter group fits [47, 48] is not straightforward since the result for $R_{b}^{0}$ of ref. [14] has been used without correspondingly modifying other $\Gamma_{q}$-related observables and without accounting for other possibly large fermionic two-loop corrections. 
Table 1. Summary of experimental data and fit results in the SM, including the subleading two-loop fermionic EW corrections to $\rho_{Z}^{f}$ with the results of ref. $[14,29]$ and introducing the parameters $\delta \rho_{Z}^{v, \ell, b}$. The values in the column "Indirect" are determined without using the corresponding experimental information. The last column shows the pulls in units of standard deviations evaluated from the p.d.f.'s of "Data" and "Indirect" as explained in ref. [33]. For completeness we also report the fit result for $\delta \rho_{Z}^{u, d}$ computed from $\delta \rho_{Z}^{b}$ using $\Gamma_{u, d} / \Gamma_{b}$.

\begin{tabular}{lcccc}
\hline & Data & Fit & Indirect & Pull \\
\hline$\alpha_{s}\left(M_{Z}^{2}\right)$ & $0.1184 \pm 0.0006$ & $0.1184 \pm 0.0006$ & $0.078 \pm 0.024$ & -1.9 \\
$\Delta \alpha_{\mathrm{had}}^{(5)}\left(M_{Z}^{2}\right)$ & $0.02750 \pm 0.00033$ & $0.02742 \pm 0.00026$ & $0.02728 \pm 0.00043$ & -0.4 \\
$M_{Z}[\mathrm{GeV}]$ & $91.1875 \pm 0.0021$ & $91.1878 \pm 0.0021$ & $91.204 \pm 0.013$ & +1.2 \\
$m_{t}[\mathrm{GeV}]$ & $173.2 \pm 0.9$ & $173.5 \pm 0.8$ & $175.7 \pm 2.6$ & +0.9 \\
$m_{h}[\mathrm{GeV}]$ & $125.6 \pm 0.3$ & $125.6 \pm 0.3$ & $98.5 \pm 27.7$ & -0.8 \\
$\delta \rho_{Z}^{v}$ & - & $-0.0052 \pm 0.0031$ & - & - \\
$\delta \rho_{Z}^{\ell}$ & - & $-0.0002 \pm 0.0010$ & - & - \\
$\delta \rho_{Z}^{b}$ & - & $-0.0021 \pm 0.0011$ & - & - \\
\hline$\delta \rho_{Z}^{u}$ & - & $0.0026 \pm 0.0012$ & - & - \\
$\delta \rho_{Z}^{d}$ & - & $0.0023 \pm 0.0012$ & - & - \\
\hline$M_{W}[\mathrm{GeV}]$ & $80.385 \pm 0.015$ & $80.366 \pm 0.007$ & $80.361 \pm 0.007$ & -1.4 \\
$\Gamma_{W}[\mathrm{GeV}]$ & $2.085 \pm 0.042$ & $2.0890 \pm 0.0006$ & $2.0890 \pm 0.0006$ & +0.1 \\
$\Gamma_{Z}[\mathrm{GeV}]$ & $2.4952 \pm 0.0023$ & $2.4952 \pm 0.0023$ & - & - \\
$\sigma_{h}^{0}[\mathrm{nb}]$ & $41.540 \pm 0.037$ & $41.539 \pm 0.037$ & - & - \\
$\sin ^{2} \theta_{\mathrm{eff}}^{\mathrm{lept}}\left(Q_{\mathrm{FB}}^{\mathrm{had}}\right)$ & $0.2324 \pm 0.0012$ & $0.23145 \pm 0.00009$ & $0.23145 \pm 0.00009$ & -0.8 \\
$P_{\tau}^{\mathrm{pol}}$ & $0.1465 \pm 0.0033$ & $0.1476 \pm 0.0007$ & $0.1476 \pm 0.0007$ & +0.3 \\
$\mathcal{A}_{\ell}(\mathrm{SLD})$ & $0.1513 \pm 0.0021$ & $0.1476 \pm 0.0007$ & $0.1470 \pm 0.0008$ & -1.9 \\
$\mathcal{A}_{c}$ & $0.670 \pm 0.027$ & $0.6681 \pm 0.0003$ & $0.6681 \pm 0.0003$ & -0.1 \\
$\mathcal{A}_{b}$ & $0.923 \pm 0.020$ & $0.93466 \pm 0.00006$ & $0.93466 \pm 0.00006$ & +0.6 \\
$A_{\mathrm{FB}}^{0, \ell}$ & $0.0171 \pm 0.0010$ & $0.0163 \pm 0.0002$ & $0.0163 \pm 0.0002$ & -0.8 \\
$A_{\mathrm{FB}}^{0, c}$ & $0.0707 \pm 0.0035$ & $0.0739 \pm 0.0004$ & $0.0740 \pm 0.0004$ & +0.9 \\
$A_{\mathrm{FB}}^{0, b}$ & $0.0992 \pm 0.0016$ & $0.1034 \pm 0.0005$ & $0.1038 \pm 0.0005$ & +2.7 \\
$R_{\ell}^{0,}$ & $20.767 \pm 0.025$ & $20.768 \pm 0.025$ & - & - \\
$R_{c}^{0}$ & $0.1721 \pm 0.0030$ & $0.17247 \pm 0.00002$ & $0.17247 \pm 0.00002$ & +0.1 \\
$R_{b}^{0}$ & $0.21629 \pm 0.00066$ & $0.21492 \pm 0.00003$ & $0.21492 \pm 0.00003$ & -2.1 \\
\hline & & & & \\
\hline & - & & - & - \\
\hline & - & & & - \\
\hline
\end{tabular}

Table 2. Same as table 1, but using the large- $m_{t}$ expansion for the two-loop fermionic EW corrections to $\rho_{Z}^{f}$.

\begin{tabular}{lcccc}
\hline & Data & Fit & Indirect & Pull \\
\hline$\alpha_{s}\left(M_{Z}^{2}\right)$ & $0.1184 \pm 0.0006$ & $0.1184 \pm 0.0006$ & $0.1193 \pm 0.0027$ & +0.3 \\
$\Delta \alpha_{\mathrm{had}}^{(5)}\left(M_{Z}^{2}\right)$ & $0.02750 \pm 0.00033$ & $0.02740 \pm 0.00026$ & $0.02725 \pm 0.00042$ & -0.5 \\
$M_{Z}[\mathrm{GeV}]$ & $91.1875 \pm 0.0021$ & $91.1878 \pm 0.0021$ & $91.197 \pm 0.012$ & +0.8 \\
$m_{t}[\mathrm{GeV}]$ & $173.2 \pm 0.9$ & $173.5 \pm 0.8$ & $176.3 \pm 2.5$ & +1.1 \\
$m_{h}[\mathrm{GeV}]$ & $125.6 \pm 0.3$ & $125.6 \pm 0.3$ & $97.3 \pm 26.9$ & -0.9 \\
\hline$M_{W}[\mathrm{GeV}]$ & $80.385 \pm 0.015$ & $80.367 \pm 0.007$ & $80.362 \pm 0.007$ & -1.4 \\
$\Gamma_{W}[\mathrm{GeV}]$ & $2.085 \pm 0.042$ & $2.0891 \pm 0.0006$ & $2.0891 \pm 0.0006$ & +0.1 \\
$\Gamma_{Z}[\mathrm{GeV}]$ & $2.4952 \pm 0.0023$ & $2.4953 \pm 0.0004$ & $2.4953 \pm 0.0004$ & +0.0 \\
$\sigma_{h}^{0}[\mathrm{nb}]$ & $41.540 \pm 0.037$ & $41.484 \pm 0.004$ & $41.484 \pm 0.004$ & -1.5 \\
$\sin ^{2} \theta_{\mathrm{eff}}^{\text {lept }}\left(Q_{\mathrm{FB}}^{\mathrm{had}}\right)$ & $0.2324 \pm 0.0012$ & $0.23145 \pm 0.00009$ & $0.23144 \pm 0.00009$ & -0.8 \\
$P_{\tau}^{\mathrm{pol}}$ & $0.1465 \pm 0.0033$ & $0.1476 \pm 0.0007$ & $0.1477 \pm 0.0007$ & +0.3 \\
$\mathcal{A}_{\ell}(\mathrm{SLD})$ & $0.1513 \pm 0.0021$ & $0.1476 \pm 0.0007$ & $0.1471 \pm 0.0008$ & -1.9 \\
$\mathcal{A}_{c}$ & $0.670 \pm 0.027$ & $0.6682 \pm 0.0003$ & $0.6682 \pm 0.0003$ & -0.1 \\
$\mathcal{A}_{b}$ & $0.923 \pm 0.020$ & $0.93466 \pm 0.00006$ & $0.93466 \pm 0.00006$ & +0.6 \\
$A_{\mathrm{FB}}^{0, \ell}$ & $0.0171 \pm 0.0010$ & $0.0163 \pm 0.0002$ & $0.0163 \pm 0.0002$ & -0.8 \\
$A_{\mathrm{FB}}^{0, c}$ & $0.0707 \pm 0.0035$ & $0.0740 \pm 0.0004$ & $0.0740 \pm 0.0004$ & +0.9 \\
$A_{\mathrm{FB}}^{0, b}$ & $0.0992 \pm 0.0016$ & $0.1035 \pm 0.0005$ & $0.1039 \pm 0.0005$ & +2.8 \\
$R_{\ell}^{0}$ & $20.767 \pm 0.025$ & $20.735 \pm 0.004$ & $20.734 \pm 0.004$ & -1.3 \\
$R_{c}^{0}$ & $0.1721 \pm 0.0030$ & $0.17223 \pm 0.00002$ & $0.17223 \pm 0.00002$ & +0.0 \\
$R_{b}^{0}$ & $0.21629 \pm 0.00066$ & $0.21575 \pm 0.00003$ & $0.21575 \pm 0.00003$ & -0.8 \\
\hline
\end{tabular}



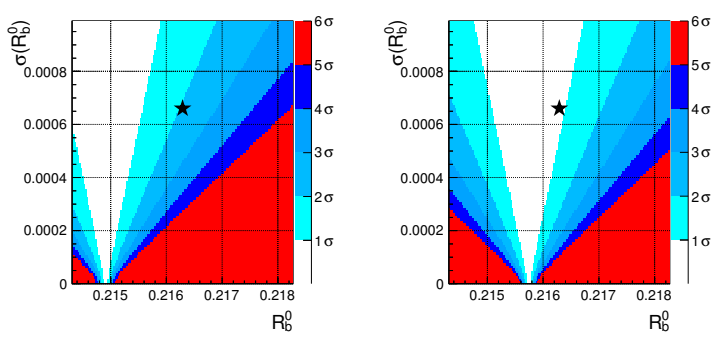

Figure 2. Compatibility plot of $R_{b}^{0}$ computed using the results of ref. [14] (left) or the large $m_{t}$ expansion for the two-loop fermionic EW corrections to $\rho_{Z}^{f}$ (right).

Let us now discuss the EW fit beyond the SM, using several widely adopted model-independent parameterizations of NP contributions. Before dwelling into the details of the different analyses, a discussion on the inclusion of the results of ref. [14, 29] is mandatory. In our SM fit, we parameterized the unknown two-loop fermionic EW corrections to $\rho_{Z}^{f}$ with three free parameters. The fit result selects values of these corrections that are as large as the ones computed by Freitas and Huang, and much larger than naively expected from the large- $m_{t}$ expansion. Waiting for a complete calculation of these corrections, we cannot use consistently the results of ref. $[14,29]$ in NP fits where the use of $R_{\ell}^{0}, \Gamma_{Z}$ and $\sigma_{h}^{0}$ is necessary to constrain NP contributions. Thus, in these cases we only present results obtained using the large- $m_{t}$ expansion for the twoloop fermionic EW corrections to $\rho_{Z}^{f}$, while in other cases we present results using both the large- $m_{t}$ expansion and the expressions in ref. [14, 29], leaving the choice of the preferred option to the reader. In the latter case, we do not use the observables $\Gamma_{Z}, R_{\ell}^{0}$ and $R_{c}^{0}$ in the fit. In all the NP fits reported below, the fit result for SM parameters practically coincides with the input reported in table 1.

In several NP scenarios, the dominant NP effects appear in the gauge-boson vacuum-polarization corrections, called oblique corrections $[49,50]$. If the NP scale is sufficiently higher than the weak scale, the oblique corrections are effectively described by the three independent parameters $S, T$ and $U[4,51]$. NP contributions to an observable, parameterized by the above oblique parameters, add up to the SM contribution:

$$
O=O_{\mathrm{SM}}+O_{N P}(S, T, U)
$$

where $S=T=U=0$ in the SM, and we linearize the NP contribution in terms of the oblique parameters [4, 51-54]. For $S, T$ and $U$ all different from zero, $\Gamma_{Z}$ is necessary to obtain bounds on the NP parameters, so in this case we only use the large- $m_{t}$ expansion. We fit the three oblique parameters together with the SM parameters to the EW precision data in table 1 . The fit results are summarized in the second column of table 3 . The two-dimensional probability distribution for $S$ and $T$ is shown in the left plot of figure 3 .

If one fixes $U=0$, which is the case in many NP models where $U \ll S, T$, the fit yields the results in the third
Table 3. Fit results for the oblique parameters with floating $U$ or fixing $U=0$, using the large- $m_{t}$ expansion or with the results of ref. $[14,29]$ for the two-loop fermionic EW corrections to $\rho_{Z}^{f}$.

\begin{tabular}{c|cc|c}
\hline & \multicolumn{2}{|c|}{ Large- $m_{t}$ expansion } & Using ref. [14, 29] \\
& $S T U$ fit & $S T$ fit with $U=0$ & $S T$ fit with $U=0$ \\
\hline$S$ & $0.04 \pm 0.10$ & $0.06 \pm 0.09$ & $0.08 \pm 0.10$ \\
$T$ & $0.05 \pm 0.12$ & $0.08 \pm 0.07$ & $0.10 \pm 0.08$ \\
$U$ & $0.03 \pm 0.09$ & - & - \\
\hline
\end{tabular}
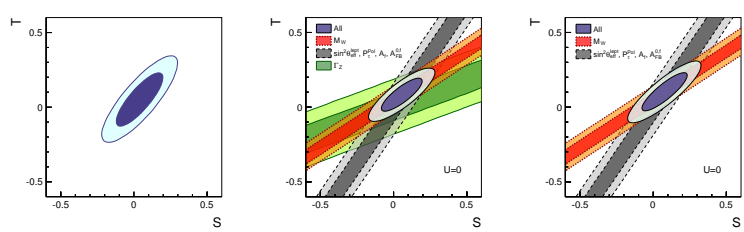

Figure 3. Left: Two-dimensional probability distribution for the oblique parameters $S$ and $T$ obtained from the fit with $S, T, U$ and the SM parameters, with the large- $m_{t}$ expansion for the twoloop fermionic EW corrections to $\rho_{Z}^{f}$. The dark (light) regions correspond to $68 \%$ (95\%) probability. Center: Two-dimensional probability distribution for the oblique parameters $S$ and $T$ obtained from the fit with $S, T$ and the SM parameters with $U=0$, with the large- $m_{t}$ expansion for the two-loop fermionic EW corrections to $\rho_{Z}^{f}$. Right: Same as center, but using the results of ref. $[14,29]$.

(fourth) column of table 3 omitting (using) the formulæ of ref. $[14,29]$. The corresponding two-dimensional distribution is given in the center and right plots in figure 3 . As expected, the results in the case $U=0$ do not depend sizably on the choice made for the two-loop fermionic EW corrections.

A key question to understand the mechanism of EWSB is whether the underlying dynamics is weak or strong. As we shall see below, EWPO strongly constrain the Higgs coupling to vector bosons, and this hints either at a weakly interacting Higgs or at a non-trivial strongly interacting sector in which additional contributions to EWPO are present and restore the agreement with experimental data. To investigate the question above, it is useful to consider a general Lagrangian for a light Higgs-like scalar field $h$ [15-18]. Under the assumption of an approximate custodial symmetry, the longitudinal $W$ and $Z$ polarizations can be described by the two-by-two matrix $\Sigma(x)=\exp \left(i \tau^{a} \chi^{a}(x) / v\right)$, with $\tau^{a}$ the Pauli matrices and $v^{2}=1 /\left(\sqrt{2} G_{\mu}\right)$. Then, assuming that there are no other light states and no new sources of flavour violation, the dominant contribution to EWPO is given by the coupling $a$ defined by $[16,17]$ :

$$
\mathcal{L}_{a}=\frac{v^{2}}{4} \operatorname{Tr}\left(D_{\mu} \Sigma^{\dagger} D^{\mu} \Sigma\right)\left(1+2 a \frac{h}{v}+\cdots\right)
$$

The SM corresponds to the choice $a=1$. The dominant deviations from the SM in EWPO are induced by the nonstandard coupling $a \neq 1$. This generates extra contribu- 

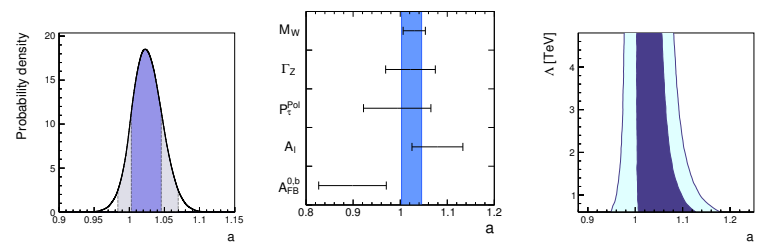

Figure 4. Left: Probability distribution for the coupling $a$. Center: Indirect determinations of the coupling $a$, excluding the observables $M_{W}, \Gamma_{Z}, P_{\tau}^{\mathrm{pol}}, A_{l}^{0}$ and $A_{\mathrm{FB}}^{0, b}$, except for the one specified in each row. The vertical blue band represents the one obtained from the the fit with all the observables. Right: Probability regions in the $a-\Lambda$ plane.

tions to the $S$ and $T$ parameters [55]:

$$
S=\frac{1}{12 \pi}\left(1-a^{2}\right) \ln \left(\frac{\Lambda^{2}}{m_{h}^{2}}\right), \quad T=-\frac{3}{16 \pi c_{W}^{2}}\left(1-a^{2}\right) \ln \left(\frac{\Lambda^{2}}{m_{h}^{2}}\right),
$$

where $\Lambda=4 \pi v / \sqrt{\left|1-a^{2}\right|}$ is the cutoff of the light Higgs effective Lagrangian. A sum rule for $1-a^{2}$ can be written in terms of the total cross sections in different isospin channels of longitudinal EW gauge boson scattering [56], implying $a^{2} \leq 1$ unless the $I=2$ channel dominates the cross section. Thus, we expect in general a positive $S$ and a negative $T$. We fit the coupling $a$ together with the five SM parameters to the precision observables using the large- $m_{t}$ expansion for the two-loop fermionic EW corrections to $\rho_{Z}^{f}$, and obtain the results shown in the left plot in figure 4 .

Since the fit prefers values of $a>1$, while the sum rule of ref. [56] gives in general $a<1$, additional contributions to the EWPO, for example from additional light fermions $[55,57]$, are required in order to restore the agreement with experimental data in composite Higgs models. If one takes literally the model with no new particles below the cutoff and assuming $a \leq 1$, from the $95 \%$ probability range $a \in[0.984,1.070]([0.981,1.071])$ one can derive a lower bound on $\Lambda$ :

$$
\Lambda>17 \text { (16) TeV@95\% probability , }
$$

using the large- $m_{t}$ expansion (using the results of ref. [14, 29]). One can generalise the analysis allowing for $\Lambda<$ $4 \pi v / \sqrt{\left|1-a^{2}\right|}$ and assuming that the dynamics at the cutoff does not contribute sizably to $S$ and $T$. In this case one can determine regions in the $a-\Lambda$ plane as shown in right plot of figure 4. Clearly the value of $a$ is tightly constrained for values of $\Lambda$ compatible with direct searches.

Before concluding, let us take a more general approach and consider the contributions to the EW fit of arbitrary dimension-six NP-induced operators $[11,18,58]$ :

$$
\mathcal{L}_{\mathrm{eff}}=\mathcal{L}_{\mathrm{SM}}+\sum_{i} \frac{C_{i}}{\Lambda^{2}} O_{i}
$$

For concreteness, let us use the operator basis of ref. [11]. For fermions, we do not consider generation mixing, and assume lepton-flavour universality: $C_{H L}^{\prime}=C_{H L i}^{\prime}, C_{H L}=$ $C_{H L_{i}}$ and $C_{H E}=C_{H E_{i}}$ for $i=1,2,3$. Switching on one operator at a time (thus barring accidental cancellations), one can constrain the coefficient of each of the above operators using the EW fit. Clearly, as is the case for all indirect constraints, one can either interpret this as a bound on the NP scale fixing the coupling or as a bound on the coupling for fixed NP scale. In table 4, we list for all the operators the $95 \%$ probability regions of the coefficients and the lower bound on the NP scale in $\mathrm{TeV}$ obtained by setting $C_{i}= \pm 1$, assuming quark-flavour universality for the operators. Results obtained relaxing the quark-flavour universality assumption, and also allowing several operators to contribute simultaneously, can be found in ref. [28]. Our fit results are also compatible with the ones of ref. [59], considering that in the latter work $m_{h}$ was not yet available and that in the fit the other SM parameters were not floated. While the results we obtained are consistent with the nonobservation of NP at the 7 and $8-\mathrm{TeV}$ runs, the possibility of weakly-interacting NP hiding behind the corner remains unscathed.

M.C. is associated to the Dipartimento di Matematica e Fisica, Università di Roma Tre, and E.F. and L.S. are associated to the Dipartimento di Fisica, Università di Roma "La Sapienza". M.C, E.F and L.S. acknowledge partial support from MIUR (Italy) under the PRIN 20102011 program. The research leading to these results has received funding from the European Research Council under the European Union's Seventh Framework Programme (FP/2007-2013) / ERC Grant Agreements n. 279972 and n. 267985.

\section{References}

[1] U. Amaldi et al., Phys.Rev. D36, 1385 (1987)

[2] G. Costa et al., Nucl.Phys. B297, 244 (1988)

[3] P. Langacker, M.x. Luo, Phys.Rev. D44, 817 (1991)

[4] M.E. Peskin, T. Takeuchi, Phys.Rev. D46, 381 (1992)

[5] J. Erler, P. Langacker, Phys.Rev. D52, 441 (1995)

[6] G. Altarelli, R. Barbieri, Phys.Lett. B253, 161 (1991)

[7] G. Altarelli, R. Barbieri, S. Jadach, Nucl.Phys. B369, 3 (1992)

[8] G. Altarelli, R. Barbieri, F. Caravaglios, Nucl.Phys. B405, 3 (1993)

[9] R. Barbieri et al., Nucl.Phys. B703, 127 (2004)

[10] B. Grinstein, M.B. Wise, Phys.Lett. B265, 326 (1991)

[11] R. Barbieri, A. Strumia, Phys.Lett. B462, 144 (1999)

[12] ATLAS Collaboration, Phys.Lett. B716, 1 (2012)

[13] CMS Collaboration (2013), 1303.4571

[14] A. Freitas, Y.C. Huang, JHEP 1208, 050 (2012)

[15] G. Giudice et al., JHEP 0706, 045 (2007)

[16] R. Contino et al., JHEP 1005, 089 (2010)

[17] A. Azatov, R. Contino, J. Galloway, JHEP 1204, 127 (2012)

[18] R. Contino et al. (2013), 1303.3876

[19] Particle Data Group, Phys.Rev. D86, 010001 (2012)

[20] S. Bethke, Nucl.Phys.Proc.Suppl. 234, 229 (2013) 
Table 4. Fit results for the coefficients of the dimension six operators at $95 \%$ probability in units of $1 / \Lambda^{2} \mathrm{TeV}^{-2}$, with quark-flavour universality in NP contribution. The fit is performed switching on one operator at a time. The corresponding lower bounds on the NP scale in $\mathrm{TeV}$ obtained by setting $C_{i}= \pm 1$ are also presented.

\begin{tabular}{c|c|cc|c|rc}
\hline & \multicolumn{3}{|c|}{ Large- $m_{t}$ expansion } & \multicolumn{2}{c}{ Using ref. [14, 29] } \\
\hline \multirow{3}{*}{ Coefficient } & $C_{i} / \Lambda^{2}\left[\mathrm{TeV}^{-2}\right]$ & \multicolumn{2}{|c|}{$\Lambda[\mathrm{TeV}]$} & $C_{i} / \Lambda^{2}\left[\mathrm{TeV}^{-2}\right]$ & \multicolumn{2}{|c}{$\Lambda[\mathrm{TeV}]$} \\
& at 95\% & $C_{i}=-1$ & $C_{i}=1$ & at 95\% & $C_{i}=-1$ & $C_{i}=1$ \\
\hline$C_{W B}$ & {$[-0.0096,0.0042]$} & 10.2 & 15.4 & {$[-0.0095,0.0045]$} & 10.3 & 15.0 \\
$C_{H}$ & {$[-0.030,0.007]$} & 5.8 & 12.1 & {$[-0.031,0.008]$} & 5.7 & 11.5 \\
$C_{L L}$ & {$[-0.011,0.019]$} & 9.5 & 7.2 & {$[-0.016,0.023]$} & 8.0 & 6.6 \\
$C_{H L}^{\prime}$ & {$[-0.012,0.005]$} & 9.2 & 14.1 & {$[-0.017,0.009]$} & 7.6 & 10.8 \\
$C_{H Q}^{\prime}$ & {$[-0.010,0.015]$} & 10.2 & 8.2 & {$[-0.40,0.20]$} & 1.6 & 2.2 \\
$C_{H L}$ & {$[-0.007,0.010]$} & 12.2 & 10.0 & {$[-0.034,0.022]$} & 5.5 & 6.7 \\
$C_{H Q}$ & {$[-0.023,0.046]$} & 6.6 & 4.7 & {$[-0.01,0.11]$} & 11.7 & 3.0 \\
$C_{H E}$ & {$[-0.014,0.008]$} & 8.4 & 11.1 & {$[-0.029,0.019]$} & 5.9 & 7.2 \\
$C_{H U}$ & {$[-0.061,0.087]$} & 4.0 & 3.4 & {$[-0.37,0.08]$} & 1.6 & 3.5 \\
$C_{H D}$ & {$[-0.15,0.05]$} & 2.6 & 4.6 & {$[-1.1,-0.2]$} & 1.0 & - \\
\hline
\end{tabular}

[21] H. Burkhardt, B. Pietrzyk, Phys.Rev. D84, 037502 (2011)

[22] CDF and D0 Collaborations, Phys.Rev. D86, 092003 (2012)

[23] ATLAS and CMS Collaborations (2012), ATLASCONF-2012-095, CMS-PAS-TOP-12-001.

[24] K. Chetyrkin, B.A. Kniehl, M. Steinhauser, Phys.Rev.Lett. 79, 2184 (1997)

[25] K. Chetyrkin, Phys.Lett. B404, 161 (1997)

[26] K. Chetyrkin, J.H. Kuhn, M. Steinhauser, Comput.Phys.Commun. 133, 43 (2000)

[27] The LEP Electroweak Working Group, http:// lepewwg.web.cern.ch/LEPEWWG/

[28] M. Ciuchini et al. (2013), 1306. 4644

[29] A. Freitas, Private communication

[30] The Tevatron Electroweak Working Group for the CDF and D0 Collaborations (2012), 1204.0042

[31] ALEPH, DELPHI, L3, OPAL, SLD Collaborations, LEP Electroweak Working Group, and SLD Electroweak and Heavy Flavour Groups, Phys.Rept. 427, 257 (2006)

[32] ALEPH, CDF, D0, DELPHI, L3, OPAL, SLD Collaborations, LEP Electroweak Working Group, Tevatron Electroweak Working Group, and SLD Electroweak and Heavy Flavour Groups (2010), 1012.2367

[33] UTfit Collaboration, JHEP 0507, 028 (2005)

[34] G. Degrassi, P. Gambino, Nucl.Phys. B567, 3 (2000)

[35] R. Barbieri et al., Phys.Lett. B288, 95 (1992)

[36] R. Barbieri et al., Nucl.Phys. B409, 105 (1993)

[37] J. Fleischer, O. Tarasov, F. Jegerlehner, Phys.Lett. B319, 249 (1993)

[38] J. Fleischer, O. Tarasov, F. Jegerlehner, Phys.Rev. D51, 3820 (1995)
[39] G. Degrassi, P. Gambino, A. Vicini, Phys.Lett. B383, 219 (1996)

[40] G. Degrassi, P. Gambino, A. Sirlin, Phys.Lett. B394, 188 (1997)

[41] D.Y. Bardin et al., Comput.Phys.Commun. 133, 229 (2001)

[42] D.Y. Bardin et al. (1992), hep-ph/9412201

[43] A. Arbuzov et al., Comput.Phys.Commun. 174, 728 (2006)

[44] A. Akhundov, A. Arbuzov, S. Riemann, T. Riemann (2013), 1302.1395

[45] J. Erler (2012), 1209. 3324

[46] O. Eberhardt et al., Phys.Rev.Lett. 109, 241802 (2012)

[47] M. Baak et al., Eur.Phys.J. C72, 2205 (2012)

[48] M. Baak, R. Kogler (2013), 1306.0571

[49] D. Kennedy, B. Lynn, Nucl.Phys. B322, 1 (1989)

[50] D. Kennedy, B. Lynn, C. Im, R. Stuart, Nucl.Phys. B321, 83 (1989)

[51] M.E. Peskin, T. Takeuchi, Phys.Rev.Lett. 65, 964 (1990)

[52] I. Maksymyk, C. Burgess, D. London, Phys.Rev. D50, 529 (1994)

[53] C. Burgess et al., Phys.Lett. B326, 276 (1994)

[54] C. Burgess et al., Phys.Rev. D49, 6115 (1994)

[55] R. Barbieri et al., Phys.Rev. D76, 115008 (2007)

[56] A. Falkowski, S. Rychkov, A. Urbano, JHEP 1204, 073 (2012)

[57] C. Grojean, O. Matsedonskyi, G. Panico (2013), 1306.4655

[58] W. Buchmuller, D. Wyler, Nucl.Phys. B268, 621 (1986)

[59] F. del Aguila, J. de Blas, Fortsch.Phys. 59, 1036 (2011); J. de Blas, talk at this conference. 